\title{
LA ADMINISTRACIÓN ESTRATÉGICA: RESPUESTA A LAS NECESIDADES ACTUALES DE LA EDUCACIÓN
}

AUTORES: Perpetua Elizabeth Lucio Alarcón ${ }^{1}$

Alber Jeovanny Ibáñez Castillo ${ }^{2}$

Cleotilde Elizabeth Giler Quiroz ${ }^{3}$

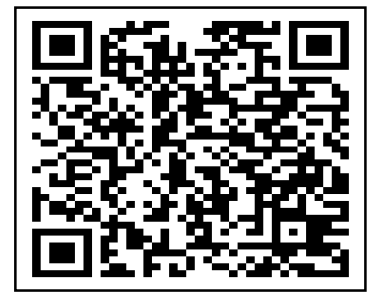

\section{DIRECCIÓN PARA CORRESPONDENCIA: (elyz7910@gmail.com)}

Fecha de recepción: 10/01/2021

Fecha de aceptación: 20/08/2021

\section{RESUMEN}

El artículo que se presenta tiene como objetivo reflexionar sobre la importancia de la administración estratégica para lograr los fines de la educación y la enseñanza. El estudio bibliográfico y el análisis respectivo realizado permite concluir que las etapas de la planificación estratégica: filosófica, analítica y operativa se constituyen en el elemento esencial de la administración estratégica para lograr los fines de la educación y la enseñanza en las condiciones actuales de la educación, lo que se complementa con los indicadores de viabilidad, de competencia y adecuación, los cuales permiten llevar a la institución a peldaños superiores con respecto a la calidad de los procesos internos y externos en pos de satisfacer las necesidades y resolver los problemas actuales.

PALABRAS CLAVE: educación, etapas, necesidades actuales y planificación estratégica.

\section{STRATEGIC MANAGEMENT: RESPONSE TO THE CURRENT NEEDS OF EDUCATION}

\section{ABSTRACT}

The article that is presented aims to reflect on the importance of strategic management to achieve the goals of education and teaching. The bibliographic study and the respective analysis carried out allows to conclude that the stages of strategic planning: philosophical, analytical and

\footnotetext{
${ }^{1}$ Máster en Gestión Educativa.Unidad Educativa Federación Deportiva de Cotopaxi Ecuador. Orcid.0000-00028010-4699

2 Ingeniero Agrónomo. Docente Independiente. La Maná, Provincia de Cotopaxi, Ecuador. Email:ajic10@hotmail.com

${ }^{3}$ Magíster en Educación Básica. Docente Independiente. La Maná, Provincia de Cotopaxi, Ecuador.Orcid,00000001-8187-2860 E-mail:cleogiler83@gmail.com
} 
operational constitute the essential element of strategic administration to achieve the ends of education and teaching in the current conditions of education., which is complemented by the indicators of viability, competence and adequacy, which allow the institution to take the institution to a higher level with respect to the quality of internal and external processes in order to satisfy needs and solve current problems.

KEYWORDS: strategic management, education, stages, current needs and strategic planning.

\section{INTRODUCCIÓN}

En los últimos años, el mundo de la enseñanza se ha visto inundado en una progresión de paradojas que ponen de manifiesto la necesidad de ayudar a los directores a alinear sus organizaciones con los requisitos previos que el mundo global les impone en la presión con la desesperación de la gestión de las tareas cotidianas que recaen sobre la escuela y que, de hecho, abordan una colección de factores reales de interrogación para las personas que tienen la tarea de controlar este tipo de establecimientos. (Beltrán, 2016)

Como resultado de las nuevas solicitudes sociales, los educadores se ven obligados a responder y ajustarse muy rápidamente, lo que a menudo implica una deficiencia de las referencias habituales de su trabajo. De este modo, el nerviosismo, la fragilidad, la falta de respuesta, pero también el afán y la responsabilidad son respuestas apasionadas que están disponibles en las reacciones de los instructores a estos cambios. En este sentido, en un entorno educativo cambiante, es fundamental no descuidar el enfoque de los sentimientos y expresiones de calidez que componen las mentalidades y discernimientos de los instructores como segmentos esenciales que deben ser pensados y creados.

La mejora de la escuela incluye el diagnóstico de las fuentes de datos, los ciclos y las necesidades de la escuela. Es decir, diagnosticar los Recursos humanos y sus capacidades, los sistemas de ayuda y mediación, los niveles de realización de los actores implicados, caracterizar la idea de las relaciones humanas, el entorno institucional, las medidas de ordenación y del consejo y los logros obtenidos. (Ramírez C. M., 2017)

En conjunto, para que un centro educativo satisfaga de forma aceptable sus capacidades y sea fructífero, necesita seguir una interacción de administración, una asociación interna y externa que permita la mejora y el cambio incesantes para lograr los objetivos normales. Una fundación educativa ofrece una formación exhaustiva a toda el área local de instrucción, que trabajará con dispositivos de administración de instrucción, para cumplir con los propósitos de educación y dar calidad en su trabajo y servicios. (Pachas, 2019). Por estas razones el presente artículo tiene como objetivo reflexionar sobre la importancia de la administración estratégica para lograr los fines de la educación y la enseñanza.

\section{DESARROLLO}

\section{Materiales y métodos}

Es totalmente esperable descubrir publicaciones de las organizaciones y en los medios de comunicación que aluden a la calidad instructiva en función de los resultados adquiridos por los 
alumnos en las pruebas externas e internas, convirtiéndose éstas en la única norma de estimación de los centros educativos en función de los ciclos lectivos que se dan en su interior y del índice de los animadores en cuestión. De ahí que se perciba como el impacto que hace concebible el logro de las habilidades y la consecución de la proyección cercana y social de la persona.

Todas las escuelas, tienen una premisa típica en el desarrollo de los destinos y se mantienen con que las asociaciones, a través de trabajos de ordenación próximos satisfactorios, pueden reconocer el futuro ideal y construirlo desde el presente, considerándolo una "situación apostada" o "situación deseada". (Armijos, 2017)

El ordenamiento pedagógico se ha convertido en uno de los instrumentos esenciales en la administración de los establecimientos educacionales situados para el cambio y la mejora perpetua del equivalente, lo que permite articular lo elaborado por las fundaciones educacionales con otras oficinas de gobierno, las cuales deben ser reguladas como un componente de la cultura jerárquica, lo que hace concebir la autosuficiencia y competencia de los ciclos de administración institucional. (Taype, 2018).

Uno de los principales problemas que busca el administrador dentro de una organización es que en las instituciones hay una propensión comprobada para que cada persona haga simplemente la base para satisfacer sus deberes. Por lo que, es desconcertante dirigir ejercicios educativos y averiguar cómo enfrentar esta prueba, donde la escuela especialmente tiene una labor principal en el cambio de la sociedad. (Ortega, 2018), esto se diferencia con lo demostrado por Morales (2016), donde muestra que el establecimiento educativo está pasando por problemas al conformar toda la documentación necesaria para el desarrollo de su oferta educativa, necesita registros fundamentales como la licencia de funcionamiento actualizada, Proyecto Educativo Institucional, Plan de Gestión de Riesgos y Código de Convivencia.

El auge de la administración estratégica se considera una reacción a las diversas necesidades y problemas actuales de la enseñanza en varios niveles. Al mismo tiempo, es una recomendación que propone una progresión de actividades, ciclos y prácticas dependientes de los diversos entornos educativos que buscan los alumnos, los instructores y los directivos de las escuelas. La sólida competencia y la complejidad general en un periodo de expansión de la globalización son precedentes iniciales para que las asociaciones centren sus esfuerzos en tratar deliberadamente sus capacidades innovadoras. En consecuencia, la experiencia de las asociaciones muestra que el mejor enfoque para lograr la capacidad de contender es a través de la innovación y el avance, un factor crítico para la competitividad.

\section{Resultados y discusión}

"La planificación estratégica es una interacción que comienza con la fundación de los objetivos organizacionales, caracteriza los procedimientos y las disposiciones para lograr estos objetivos, y crea diseños minuciosos para garantizar la ejecución de los sistemas y, por lo tanto, obtener los acabados ideales".(Hernández, 2016, pág. 10)

Teniendo todo en cuenta, se podría decir que la planificación estratégica es un ciclo que permite diseccionar el presente y emprender lo que podría ser el destino de las asociaciones. Bajo esta 
idea, para completar el ordenamiento esencial de los establecimientos educativos, es importante contar con la responsabilidad y contribución de la relativa multitud de animadores del área local educativa; es decir, cada uno de los individuos que trabajan en la organización, desde el conserje hasta el ministro o la alta administración asumiendo alguno, así como contar con las reglas y valoraciones de la sociedad.

Necesitamos supervisar el cambio y no permitir que el cambio nos supervise, la planificación estratégica nos permite rastrear la manera correcta de enfrentarnos a los cambios consistentes que pide este mundo globalizado, para enfrentarnos a la sociedad de la información y los datos, es un instrumento que permite a los centros educativos correr tras la mejora constante y la grandeza instructiva, buscando el progreso y el avance.(Campozano, 2016, pág. 25)

En la organización Educativa los ciclos de preparación, el consejo, la observación y la evaluación (control) son significativos, percibidos como medidores o punteros para la escuela de los estudiantes, construyendo uno de los extraordinarios compromisos gerenciales, que permiten tener un sueño de la circunstancia a controlar. La formación en su simplificación excesiva puede ser considerada como una maravilla monetaria. Es importante poner recursos en el área Educativa para que sea esencial para un ciclo de mejora. Una especulación que depende de una planta real, de la capacidad humana, de los proyectos y de las diferentes necesidades.

En esta situación, la naturaleza de la instrucción se convierte en clave para la realización de los centros educativos; así, "la ola planificación estratégica es la extensión que permite conectar la circunstancia actual con el futuro ideal, en definitiva, ajustar el ser y el hacer al deber ser de las organizaciones educativas". (López, 2015, pág. 16)

Para decirlo claramente, la planificación estratégica permitirá a los centros de enseñanza elaborar una técnica para alcanzar sus objetivos y crear una ventaja. "La ventaja se concibe generalmente a partir del valor que una organización descubre cómo hacer para sus clientes y que compensa los gastos de hacerlo".

En resumen, es importante que las organizaciones educativas actuales elaboren medidas de administración esenciales que les permitan hacer frente a las necesidades del futuro. "La medida de administración estratégica es la disposición de responsabilidades, elecciones y actividades necesarias para que una organización logre una intensidad clave o rendimientos más normales".(Valle, 2017, pág. 27)

Percibir que el trabajo de los directores de escuela no es sencillo, pero tampoco es descabellado. En consecuencia, es una prueba para el director de la escuela como un pionero de la característica y el poder de sus educadores hacia la responsabilidad de educación. De este modo, se puede lograr una educación más segura y exitosa con los alumnos.

En una posición de autoridad se tiene la capacidad de hacer cambios de acuerdo con las modas recientes, por lo que se necesita romper con los modelos ideales habituales y poner a prueba nuevos planes de trabajo esenciales. Las instituciones educativas deben proponer nuevas metodologías de educación, programas de anticipación y mediación y planes educativos adecuados para fomentar los componentes defensivos individuales y cuya razón de ser no es bromear aún con los elementos para contrarrestar el efecto de las ocasiones vitales perturbadoras.

Para lograr lo anteriormente expresado deben apoyarse en las diferentes etapas de la planificación estratégica que se presentan a continuación: La etapa filosófica es la que muestra el camino,

138 UNESUM-Ciencias. Publicación cuatrimestral. Vol. 5, Año 2021, No. 5 (Septiembre-Diciembre) 
decide el rumbo esencial del establecimiento a través de la afirmación de la misión, la visión, las normas corporativas, los arreglos y las técnicas institucionales, es decir es la etapa que impone una cultura y la forma en que han de trabajar los actores de la comunidad educativa con la finalidad de alcanzar los objetivos trazados.(Valdés, 2015, pág. 47). Diríamos que la visión es el destino ideal de la organización instructiva a medio y largo plazo. (García G. J., 2020, pág. 54)

Las normas corporativas constituyen las reglas que conforman el modo de vida de un establecimiento y son el conjunto de cualidades y convicciones que dirigen la manera en que los individuos del área local de instrucción deben actuar para lograr los objetivos institucionales; en su mayor parte son impuestas por la administración superior. Como ya se ha dicho, la técnica es el camino, el manual a seguir para lograr los objetivos del centro. Los enfoques son las reglas para cumplir con el procedimiento de la organización.

La etapa analítica permite estudiar la situación actual de las organizaciones de enseñanza y esbozar el mejor enfoque para lograr el futuro ideal. Por ello, es importante desglosar los factores internos y externos que, de una forma u otra, están influyendo, de forma decidida o adversa, en la mejora ordinaria de la administración de las instituciones.(Díaz, 2015, pág. 32)

El análisis interno permite descubrir las cualidades y deficiencias que pueden influir en la administración de la institución y en la capacidad de atender a los clientes, permite hacer una descripción global de cada uno de los espacios de la organización educativa: curricular, monetario, directivos, grupos humano y otros.

En el ámbito curricular se deben analizar las fortalezas y debilidades de la planificación curricular y su gestión en (Mendoza, 2018)el proceso educativo. (Rosati, 2017, pág. 7)

- En el ámbito financiero y de gestión, se analizará las fortalezas y debilidades relacionadas con la situación financiera de la institución y su gestión.

- El ámbito de infraestructura y equipamiento está relacionado con el análisis de las fortalezas y debilidades de la infraestructura, tecnología y ubicación física etc. que la institución utiliza en las medidas de enseñanza aprendizaje.(Rosillón, 2015, pág. 42)

- El ámbito del equipo humano, es el análisis de todas las fortalezas y debilidades del recurso humano, que son los actores principales del proceso educativo, estos son: estudiantes, profesores, padres de familia y autoridades. entre otros. (Martín, 2021, pág. 13)

El análisis externo comprende la concentración de cada uno de los poderes sociales que influyen bien o negativamente en la administración de la organización educativa y que son en su mayoría delegados: monetario, político, social, mecánico, geográfico, competitiva, de segmento y social. Gracias a la investigación de los poderes sociales, se adquieren los posibles peligros que de una u otra manera están influyendo en el ciclo de administración esencial del establecimiento.

Cuando se han distinguido los objetivos estratégicos, la siguiente etapa es la planificación operativa o momentánea, que comprende la decisión del plan de actividades, los objetivos, planes de gastos funcionales, control y la observación de la planificación estratégica, que debe ser posible mediante los marcadores de administración, o el cuadro de mando integral.(Muñoz, 2021, pág. 17) 
La planeación operativa consiste en establecer claramente la ejecución de la ordenación esencial según lo indicado por los objetivos explícitos, incluye la realización de una progresión de tareas dentro de un período de tiempo determinado para lograr los objetivos y se apoya de indicadores de gestión que, además de ser un instrumento de ayuda a la dinámica, los marcadores permiten detallar sistemas y actividades para la mejora de los ciclos institucionales; además, ayudan a la evaluación de la administración educativa. En los centros de enseñanza, se pueden planificar algunos tipos de indicadores, entre los que cabe destacar los siguientes: indicadores de viabilidad, de competencia y de adecuación. (Bolívar, 2021, pág. 36)

- Los indicadores de eficacia permiten medir el nivel de consecución de los objetivos establecidos.

- Los indicadores de eficiencia permiten medir el nivel de consecución de los objetivos establecidos, pero con la menor utilización de los recursos disponibles.

- Los indicadores de efectividad integran la eficacia y la eficiencia y muestran la relación entre los recursos utilizados y el impacto obtenido.

\section{CONCLUSIONES}

Las etapas de la planificación estratégica: filosófica, analítica y operativa se constituyen en el elemento esencial para lograr los fines de la educación y la enseñanza en las condiciones actuales de la educación, lo que se complementa con los indicadores de viabilidad, de competencia y adecuación, los cuales permiten llevar a la institución a peldaños superiores con respecto a la calidad de los procesos internos y externos.

\section{REFERENCIAS BIBLIOGRÁFICAS}

Armijos, L. (2017). La prospectiva estratégica como herramienta de planificación en Instituciones de Educación Superior de Latinoamérica. Ecuador: Universidad de las Fuerzas Armadas ESPE. Obtenido de https://www.researchgate.net/profile/Lorenzo-

Beltrán, S. M. (2016). La gestión directiva: un concepto construido desde las comprensiones de los directivos docentes de las escuelas públicas bogotanas. Colombia: Revista Iberoamericana para la Investigación y el Desarrollo Educativo. doi:2007-7467

Bolívar, O. E. (2021). Gestión de la calidad educativa y clima institucional en el Instituto de Educación Superior Pedagógico Publico "Puquio", Ayacucho, 2020. Peru: Universidad César Vallejo. Obtenido de https://repositorio.ucv.edu.pe/bitstream/handle/20.500.12692/61883/Bol\%c3\%advar_OEOMSD.pdf?sequence=1\&isAllowed $=\mathrm{y}$

Campozano, V. (2016). Planificación estratégica de tecnologías de la información y comunicación. Ecuador: Revista Cientifica Dominio de las ciencias. doi:2477-8818

García, G. J. (2020). La filosofía de la educación ante la emergencia tecnológica : hacia una ética de la anticipación en tiempos de hiperconectividad. Italia: torrossa. Obtenido de https://www.torrossa.com/en/resources/an/4702340

García, M. J. (2018). Gestión curricular en centros educativos costarricenses: Un análisis desde la percepción docente y la dirección. Costa Rica: Revista Electrónica Educare. doi:1409-4258

Hernández, N. B. (2016). Universidad y Planificación Estratégica en el Ecuador. Ecuador: dialnet. doi:2224-2643

López, J. G. (2015). La gestión de los riesgos de planificación estratégica en las instituciones de educación superior. Cuba: scielo. Obtenido de 0257-4314 
Martín, F. (2021). Diagnóstico interno institucional 2020 : momento analítico-descriptivo para un enfoque del TEC hacia resultados. Costa Rica: Instituto Tecnológico de Costa Rica. Obtenido de https://repositoriotec.tec.ac.cr/handle/2238/12472

Mendoza, M. L. (2018). Uso de las tic como estrategia de mediación para el aprendizaje de la lectura en educación primaria. Colombia: Revista gestión, Competitividad e Innovación. Obtenido de https://pca.edu.co/editorial/revistas/index.php/gci/article/view/35

Morales, Z. G. (2016). La Planificación Estratégica En La Administración Educativa Para Diseño De Plan De Seguimiento. Ecuador: Universidad De Guayaquil. Obtenido de http://repositorio.ug.edu.ec/bitstream/redug/32042/1/SALVADOR\%20MORALES\%20ZULEMA.pdf

Muñoz, J. G. (2021). La lectoescritura como capacidad cognitiva: procesos componentes. Intervención en educación primaria. $\quad$ España: $\quad$ Obtenido de https://southfloridapublishing.com/ojs/index.php/jdev/article/view/219

Ortega, S. M. (2018). La gestión educativa como factor asociado en la eficacia del clima organizacional en el Colegio Fiscal Compensatorio "31 de Octubre” del cantón Samborondón, provincia del Guayas. Ecuador: Universidad Andina Simón Bolívar. Obtenido de https://repositorio.uasb.edu.ec/bitstream/10644/6376/1/T2717-MGE-Tutiven-La\%20gestion.pdf

Pachas, H. J. (2019). La planificación estratégica y su importancia en las instituciones educativas. Perú: Universidad Inca Garcilaso de la Vega Facultad de Educación. Obtenido de http://168.121.45.184/bitstream/handle/20.500.11818/4669/TRABSUFICIENCIA_PACHAS_JESSICA.pdf ?sequence $=1$ \&isAllowed $=\mathrm{y}$

Ramírez, C. M. (2017). La planificación estratégica y su relación con la eficacia de los planes de trabajo en la Institución Educativa San Roque de Castrovirreyna, año 2009. Ecuador: Universidad Nacional de Educación Enrique Guzmán y Valle. Obtenido de http://repositorio.une.edu.pe/handle/UNE/3087

Rosati, G. (2017). El Qualitative Comparative Analysis (QCA) como herramienta analítica : Dos aplicaciones para el análisis de entrevistas. Argentina: Universidad Nacional de La Plata. Facultad de Humanidades y Ciencias de la Educación. doi:http://dx.doi.org/10.24215/18537863e018

Rosillón, N. (2015). Análisis financiero: una herramienta clave para una gestión financiera eficiente. Venezuela: Revista Venezolana de Gerencia. Obtenido de http://ve.c.org/scielo.php?script=sci_arttext\&pid=S131599842009000400009

Taype, P. A. (2018). Planificación Estratégica y Gestión Institucional en las Instituciones Educativas del Nivel Secundario de la Red Educativa de Ayaccocha Acoria, 2018. Peru: Escuela de Posgrado Universidades Cesar Vallejo. Obtenido de https://repositorio.ucv.edu.pe/bitstream/handle/20.500.12692/31379/acevedo_tp.pdf?sequence=1

Valdés, D. H. (2015). Filosofía para niños y lo que significa una educación filosófica. Colombia: revistasojs. Obtenido de https://revistasojs.ucaldas.edu.co/index.php/discusionesfilosoficas/article/view/549

Valle, O. A. (2017). Planificación estratégica como instrumento de la sostenibilidad ambiental en Pymes de Barranquilla Colombia. Colombia: Universidad de la Costa. Obtenido de http://hdl.handle.net/11323/4601 
142 UNESUM-Ciencias. Publicación cuatrimestral. Vol. 5, Año 2021, No. 5 (Septiembre-Diciembre) 